hep-ph/9809575

LA-UR-98-4299

\title{
Interpretation of $D(2637)$ from heavy quark symmetry
}

\author{
Philip R. Page* \\ Theoretical Division, Los Alamos National Laboratory, \\ P.O. Box 1663, Los Alamos, NM 87545, USA
}

\begin{abstract}
We demonstate from heavy quark symmetry that the width of $D(2637)$ claimed by the DELPHI Collaboration is inconsistent with any bound state with one charm quark predicted in the $D(2637)$ mass region, except possibly $D_{3}^{*}, D_{2} j_{q}=\frac{5}{2}$ or $D^{\prime}$. The former two possibilities are favoured by heavy quark mass relations.
\end{abstract}

Keywords: radial excitation, DELPHI, heavy quark symmetry, decay, node PACS number(s): 11.30.Hv 13.25.Es 13.25.Ft 14.40.Ev 14.40.Lb

\section{Introduction}

The DELPHI Collaboration recently presented evidence for a new state $D(2637)$ at $2637 \pm$ $2 \pm 6 \mathrm{MeV}$ with a width of $<15 \mathrm{MeV}$ at $95 \%$ confidence [1]. A signal of $66 \pm 14$ events was detected, a $4.7 \sigma$ effect. The state was observed decaying to $D^{*+} \pi^{+} \pi^{-}$. However, its existence has not been confirmed by the CLEO and OPAL Collaborations in the same decay channel [2]. Moreover, there is no evidence for $D(2637)$ in $D^{*} \pi$ [1, 2].

*E-mail: prp@lanl.gov 
In this Report we shall assume the validity of the DELPHI claim, and provide theoretical interpretations for $D(2637)$, based on two assumptions:

1. The validity of lowest order heavy quark symmetry decay relations between the $K-$ meson and corresponding $D$-meson systems.

2. The validity of experimental data on the established $K$-mesons $K^{*}(1410), K^{*}(1680)$, $K_{0}^{*}(1430), K_{1}(1400)$ and $K_{3}^{*}(1780)$ [3].

These are the only assumptions that will be made, unless otherwise indicated. The latter assumption is inevitable given that no new data is likely to be forthcoming soon. The former assumption is needed to make extrapolations, independent of detailed dynamical models, from known $K$-meson decays to unknown $D$-meson decays. Although not always good within the simplest form factor assumptions (see below), it has been argued to be qualitatively valid [4] in an analysis of $K_{1}(1270)$ and $K_{2}^{*}(1430)$ [5, 6]. A specific example of how heavy quark symmetry for strange quarks gives predictions in the right ballpark is as follows. The D- to S-wave width ratio for $K_{1}(1400) \rightarrow K^{*} \pi$ is $0.04 \pm 0.01$ [7] (heavy quark symmetry predicts zero [4]); and for $K_{1}(1270) \rightarrow K^{*} \pi$ is $1.0 \pm 0.7$ [7] (heavy quark symmetry predicts infinity [四).

The radial excitation of the $D^{*}$, referred to as $D^{*^{\prime}}$, is predicted to have a mass of 2640 $\mathrm{MeV}$ in a model which predicted the $D_{2}^{*}(2460)$ mass within $40 \mathrm{MeV}$ of experiment [8]; and $2629 \mathrm{MeV}$ in a recent model which is in agreement within $20 \mathrm{MeV}$ for the observed charm orbital states [9]. The noticeable proximity of these potential model predictions to the mass of $D(2637)$ leads DELPHI to identify it as $D^{*^{\prime}}$, and hence as $J^{P}=1^{-}$. However, there are several experimentally unobserved conventional mesons which can also decay to $D^{*} \pi \pi$ and are expected in potential models in the vicinity of $D(2637)$. Of these, the radially excited $D$ with $J^{P}=0^{-}$, referred to as $D^{\prime}$, would be nearest in mass at $2580 \mathrm{MeV}$ [8 or $2579 \mathrm{MeV}$ [9]. Next nearest would be the $D_{1 j_{q}=\frac{1}{2}}^{*}$, a possibly observed [10 $1^{+}$at $2460 \mathrm{MeV}$ [ 8501 $\mathrm{MeV}$ [9] or $2585 \mathrm{MeV}$ [11]; and the $0^{+} D_{0}^{*}$ at $2400 \mathrm{MeV}$ [8], $2438 \mathrm{MeV}$ [9] or $2554 \mathrm{MeV}$ [11]. The $1^{-} D_{1 j_{q}=\frac{3}{2}}$ is at $2820 \mathrm{MeV}$ [8]. The $3^{-} D_{3}^{*}$ should be at $2830 \mathrm{MeV}$ [ 8 ] or $2760 \pm 70$ $\mathrm{MeV}$ 12]. The $2^{-}$states $D_{2 j_{q}=\frac{5}{2}}$ and $D_{2 j_{q}=\frac{3}{2}}$ are within $20 \mathrm{MeV}$ of $D_{3}^{*}$ [8]. Given the small error bars of the DELPHI mass measurement, all interpretations except $D^{*^{\prime}}$ fail on mass grounds. 
The purpose of this Report is to check which of the preceding possibilities can reproduce the tiny total width of $<15 \mathrm{MeV}$ claimed by DELPHI, assuming them to have the mass of $D(2637)$. The masses of all experimentally known states will be taken from the PDG [7].

For a given heavy-light meson with total angular momentum $\vec{J}$, let $\vec{s}_{Q}\left(s_{Q}=\frac{1}{2}\right)$ be the spin of the heavy quark and $\vec{\jmath}_{q}$ the total angular momentum of the light degrees of freedom. Consider the decay of a heavy-light meson characterized by $J, j_{q}$ to an outgoing heavy-light meson characterized by $J^{\prime}, j_{q}^{\prime}$ and a light meson with spin $s_{h}$. The light meson has orbital angular momentum $\ell$ relative to the outgoing heavy-light meson. The decay amplitude satisfies certain symmetry relations because the decay dynamics become independent of the heavy quark spin in the heavy quark limit of QCD [13]. The two-body decay width can be factored into a reduced form factor multiplied by a normalized $6-j$ symbol [13]

$$
\Gamma=\left(\sqrt{\left(2 J^{\prime}+1\right)\left(2 j_{q}+1\right)}\left\{\begin{array}{ccc}
s_{Q} & j_{q}^{\prime} & J^{\prime} \\
j_{h} & J & j_{q}
\end{array}\right\}\right)^{2} p^{2 \ell+1} F_{j_{h} \ell^{j_{j}} j_{q}^{\prime}}^{\prime}\left(p^{2}\right)
$$

where $\overrightarrow{\mathrm{J} h} \equiv \vec{s}_{h}+\vec{\ell}$. The $6-j$ symbols are evaluated in ref. [14. $p$ is the magnitude of the three-momentum of the decay products in the rest frame of the initial state. Eq. [1 neglects corrections to the heavy quark limit, except in as far as they modify $p$. One essential idea of the heavy quark limit is that the spin of the heavy quark and the total angular momentum of the light degrees of freedom are seperately conserved [13], i.e. $\overrightarrow{\jmath_{q}}=\overrightarrow{\mathrm{J}_{q}^{\prime}}+\overrightarrow{\mathrm{\jmath}} h$. This conservation law is in addition to the usual conditions of conservation of total angular momentum $\vec{J}=\vec{J}^{\prime}+\overrightarrow{\mathrm{J} h}$ and parity. For the remainder of this Report we shall restrict to $\ell$ allowed by all these conservation conditions. Heavy quark symmetry does not predict the magnitude and functional dependence of the reduced form factor $F_{j_{h} \ell}^{j_{q} \ell_{q}^{\prime}}\left(p^{2}\right)$ for a particular decay. Once determined from experimentally well established decays of $K$-mesons with given $j_{q}, j_{q}^{\prime}$, this quantity may be used to predict related decays of both $K$ - and $D$-mesons with the same $j_{q}, j_{q}^{\prime}$.

\section{Interpreting $D(2637)$ : Gaussian form factor}

We shall assume a Gaussian form for the reduced form factor [5] 
Table 1: Widths of $D(2637)$ to $D \pi$ and $D^{*} \pi$ in $\mathrm{MeV}$. The interpretation and $j_{q}$ of $D(2637)$ is given in the first and third columns respectively. Blank entries are identical to those above them. Since there are reasons to doubt that $K^{*}(1410)$ is the radially excited $K^{*}$, the $K^{*}(1680)$ is often taken to be the radial excitation [7, 18]. Another quark model interpretation of $K^{*}(1680)$ is as a D-wave meson [7, 18], so that $j_{q}=\frac{3}{2}$. The only interpretation of $K_{0}^{*}(1430)$ is as a $\mathrm{P}$-wave meson [7], so that $j_{q}=\frac{1}{2}$.

\begin{tabular}{|c|l|r|r|r|r|}
\hline$D(2637)$ & K-meson data used [20] & $j_{q}$ & Form Factor & $D \pi$ & $D^{*} \pi$ \\
\hline$D^{*^{\prime}}$ & $\Gamma\left(K^{*}(1410) \rightarrow K \pi\right)=15 \mathrm{MeV}$ & $\frac{1}{2}$ & $F_{11}^{\frac{1}{2} \frac{1}{2}}(0)$ & 17 & 22 \\
& $\Gamma\left(K^{*}(1680) \rightarrow K \pi\right)=125 \mathrm{MeV}$ & & & 86 & 115 \\
& $\Gamma\left(K^{*}(1680) \rightarrow K^{*} \pi\right)=96.3 \mathrm{MeV}$ & & & 53 & 71 \\
$D^{\prime}$ & $\Gamma\left(K^{*}(1410) \rightarrow K \pi\right)$ & & & - & 33 \\
& $\Gamma\left(K^{*}(1680) \rightarrow K \pi\right)$ & & & - & 172 \\
& $\Gamma\left(K^{*}(1680) \rightarrow K^{*} \pi\right)$ & & - & 106 \\
$D_{0}^{*}$ & $\Gamma\left(K_{0}^{*}(1430) \rightarrow K \pi\right)=270 \mathrm{MeV}$ & & $F_{00}^{\frac{1}{2} \frac{1}{2}}(0)$ & 270 & - \\
$D_{1 j_{q}=\frac{1}{2}}^{*}$ & & & & - & 260 \\
$D_{1 j_{q}=\frac{3}{2}}$ & $\Gamma\left(K^{*}(1680) \rightarrow K \pi\right)$ & $F_{11}^{\frac{3}{2} \frac{1}{2}}(0)$ & 87 & 29 \\
& $\Gamma\left(K^{*}(1680) \rightarrow K^{*} \pi\right)$ & & & 213 & 71 \\
$D_{2 j_{q}=\frac{3}{2}}$ & $\Gamma\left(K^{*}(1680) \rightarrow K \pi\right)$ & & - & 86 \\
& $\Gamma\left(K^{*}(1680) \rightarrow K^{*} \pi\right)$ & & - & 212 \\
\hline
\end{tabular}

$$
F_{j_{h} \ell}^{j_{q} j_{q}^{\prime}}\left(p^{2}\right)=F_{j_{h} \ell}^{j_{q} j_{q}^{\prime}}(0) \exp \left(-\frac{p^{2}}{6 \beta^{2}}\right)
$$

in this section. The Gaussian form arises in decay models where simple harmonic oscillator wave functions are used [15, 16, 17], and the value $\beta=0.4 \mathrm{GeV}$ is phenomenologically successful [5, 6, 15, 16, 17]. We shall adopt this value, although our predictions are stable under the variation $\beta=0.35-0.45 \mathrm{GeV}$. Tables 1 and 2 indicate the interpretations of $D(2637)$ that will be explored.

The first entry in Table 1 will be discussed in detail to clarify the methods used. For our heavy quark symmetry analysis it is not neccesary to know the nature of $K^{*}(1410)$, only the value of $j_{q}$, which can be $\frac{1}{2}$ or $\frac{3}{2}$ since $J=1$. We shall motivate our choice of $j_{q}$ from the known quark model interpretation. The only interpretation of $K^{*}(1410)$ is as a radially 
Table 2: Partial widths of $D_{3}^{*}$ and $D_{2} j_{q}=\frac{5}{2}$ in $\mathrm{MeV}$. Blank entries are identical to those above them. In some form factors we have explicitly indicated the light meson $\eta$ or $\rho$, in order to distinguish them from form factors for $\pi$. The only quark model interpretation of $K_{3}^{*}(1780)$ is as a D-wave meson [7, 18], so that $j_{q}=\frac{5}{2}$. Decays of $D(2637)$ to $D \omega, D^{*} \rho$ are below threshold by more than half a width of $\omega$ and $\rho$ respectively, and are not calculated in this Report. However, $D_{2 j_{q}=\frac{5}{2}} \rightarrow D \omega$ and $D_{3}^{*}, D_{2 j_{q}=\frac{5}{2}} \rightarrow D^{*} \rho$ can be in $\mathrm{P}$-wave and hence competitive with the rates in the text, although current experimental data on $\mathrm{K}$-mesons do not give sufficient information to estimate these rates from heavy quark symmetry. $D_{2 j_{q}=\frac{5}{2}} \rightarrow D_{0}^{*} \pi$ is a D-wave decay and $D_{3}^{*}, D_{2 j_{q}=\frac{5}{2}} \rightarrow D_{1 j_{q}=\frac{1}{2}}^{*} \pi$ a D-wave decay at threshold, using the $D_{1 j_{q}=\frac{1}{2}}^{*}$ and $D_{0}^{*}$ masses of ref. [9]. These decays cannot be estimated from experimental data. $\dagger$ Assuming SU(3) symmetry. $\ddagger$ This is an F-wave decay at threshold, and hence very sensitive to phase space. We smear the partial width (Eqs. 11 and 2) over a relativistic Breit-Wigner form to take account of the $150 \mathrm{MeV}$ width of the $\rho$. \& This decay involves form factors which cannot be estimated from experimental data. $\amalg$ The width of $D_{2}^{*}(2460)$ has been smeared over.

\begin{tabular}{|l|c|c|r|r|}
\hline$K$-meson data used [20] & Form Factor & Decay Mode & $D_{3}^{*}$ & $D_{2 j_{q}=\frac{5}{2}}$ \\
\hline$\Gamma\left(K_{3}^{*}(1780) \rightarrow K \pi\right)=29.9 \mathrm{MeV}$ & $F_{3}^{\frac{5}{2} \frac{1}{2}}(0)$ & $D \pi$ & 7.8 & - \\
& & $D^{*} \pi$ & 3.4 & 5.9 \\
$\Gamma\left(K_{3}^{*}(1780) \rightarrow K^{*} \pi\right)=32 \mathrm{MeV}$ & & $D \pi$ & 22 & - \\
& & $D^{*} \pi$ & 7.8 & 17 \\
$\Gamma\left(K_{3}^{*}(1780) \rightarrow K \pi, K^{*} \pi\right)$ & & $D_{s} K \dagger$ & $<.6$ & - \\
& & $D_{s}^{*} K \dagger$ & $\sim 0$ & $\sim 0$ \\
$\Gamma\left(K_{3}^{*}(1780) \rightarrow K \eta\right)=48 \mathrm{MeV}$ & $F_{3}^{\frac{5}{2} \frac{1}{2} \eta}(0)$ & $D \eta$ & 2.9 & - \\
& & $D^{*} \eta$ & 0.1 & 0.2 \\
$\Gamma\left(K_{3}^{*}(1780) \rightarrow K \rho\right)=49 \mathrm{MeV}$ & $F_{3}^{\frac{5}{2} \frac{1}{2} \rho}(0)$ & $D \rho \ddagger$ & 0.7 & $\mathbf{9}$ \\
$\Gamma\left(K_{3}^{*}(1780) \rightarrow K_{2}^{*}(1430) \pi\right)<25 \mathrm{MeV}$ & $F_{2}^{\frac{5}{2} \frac{3}{2}}(0)$ & $D_{2}^{*}(2460) \pi \amalg$ & $<0.5$ & $<0.2$ \\
& & $D_{1}(2420) \pi$ & $<0.2$ & $<1.1$ \\
\hline
\end{tabular}


excited $K^{*}[\overline{7}, 18]$, so that $j_{q}=\frac{1}{2}$. However, $K^{*}(1410)$ may have a non-conventional-meson component, e.g. a low-lying $1^{-}$hybrid meson with $j_{q}=\frac{1}{2}$. Noting that the $\pi$ has $s_{h}=0$, we deduce from Eqs. 11 and 2, using the experimental data on $\Gamma\left(K^{*}(1410) \rightarrow K \pi\right)$, the value of $F_{1}^{\frac{1}{2} \frac{1}{2}}(0)$. From this $\Gamma\left(D^{*^{\prime}} \rightarrow D \pi, D^{*} \pi\right)$ is calculated. The total width of $D^{*^{\prime}}$ is found to be appreciably higher than the DELPHI value. The same holds true for all other possibilities explored in Table 1.

$K_{3}^{*}(1780)$ has been used in an analogous study to the one in this Report [5, 6]. There the heavy quark symmetry partners $D_{3}^{*}$ and $D_{2} j_{q}=\frac{5}{2}$ have been found to be $193 \mathrm{MeV}$ and 99 $\mathrm{MeV}$ wide [6], respectively, due to the high mass of $2830 \mathrm{MeV}$ used [19]. In this work we use the mass of the $D(2637)$ by fiat, so that the total widths should be substantially smaller.

The partial widths of $D_{3}^{*}$ are estimated in Table 2 . All decay modes other than $D \pi$ and $D^{*} \pi$ contributes $4-5 \mathrm{MeV}$. The $D \pi$ and $D^{*} \pi$ partial widths depend on which $\mathrm{K}$-meson decay they are fixed to. Fixing from $K_{3}^{*}(1780) \rightarrow K \pi$, a partial width with a small experimental uncertainty [20], yields a total $D_{3}^{*}$ width of $15-16 \mathrm{MeV}$. Fixing from $K_{3}^{*}(1780) \rightarrow K^{*} \pi$ has the advantage that the dominant decay $D_{3}^{*} \rightarrow D \pi$ has almost exactly the same momentum $p$, so that $\Gamma\left(D_{3}^{*} \rightarrow D \pi\right) / \Gamma\left(K_{3}^{*}(1780) \rightarrow K^{*} \pi\right)=3 / 4$ from heavy quark symmetry independent of the assumed form factor. Here the total $D_{3}^{*}$ width is $36-37 \mathrm{MeV}$. Since we have not estimated $D_{3}^{*} \rightarrow D^{*}(\pi \pi)_{S}$ due to lack of experimental data from $\mathrm{K}$-mesons it appears likely that $D_{3}^{*}$ cannot be interpreted as $D(2637)$ based on its total width, although the possibility cannot be eliminated.

The decays of $D_{2 j_{q}=\frac{5}{2}}$ are also estimated in Table 2. The total estimated width of $D_{2} j_{q}=\frac{5}{2}$ is $6-7$ or $17-18 \mathrm{MeV}$ depending on whether we fix respectively from $K_{3}^{*}(1780) \rightarrow K \pi$ or $K_{3}^{*}(1780) \rightarrow K^{*} \pi$. Since we cannot estimate $D_{2 j_{q}=\frac{5}{2}} \rightarrow D(\pi \pi)_{S}, D^{*}(\pi \pi)_{S}$, which have substantial phase space, the balance of probability is that the total $D_{2 j_{q}=\frac{5}{2}}$ width is not consistent with the DELPHI value.

The conclusion of this section is that all interpretations of $D(2637)$ have too large total widths, except possibly $D_{3}^{*}$ and $D_{2} j_{q}=\frac{5}{2}$, of which $D_{2 j_{q}=\frac{5}{2}}$ appears to be the narrowest candidate. 


\section{Interpreting $D(2637)$ : Nodal Gaussian form factor}

Based on the ${ }^{3} P_{0}$ model decay amplitude, we postulate the nodal Gaussian form factor 16, 17

$$
F_{j_{h} \ell}^{j_{q} j_{q}^{\prime}}\left(p^{2}\right)=F_{j_{h} \ell}^{j_{q} j_{q}^{\prime}}(0)\left(1-\frac{2}{15} \frac{p^{2}}{\tilde{\beta}^{2}}\right)^{2} \exp \left(-\frac{p^{2}}{6 \beta^{2}}\right)
$$

at the cost of introducing an extra parameter $\tilde{\beta}$. The experimental motivation for this form factor is that the experimental ratio $\Gamma\left(K^{*}(1410) \rightarrow K \pi\right) / \Gamma\left(K^{*}(1410) \rightarrow K^{*} \pi\right)<0.16$ [7] is at least a factor of eight smaller than the heavy quark symmetry prediction with a Gaussian form factor ${ }^{\prime}$. This indicates the need for a form factor which can additionally suppress $K^{*}(1410) \rightarrow K \pi$. The theoretical motivation is that the radially excited $K^{*}(1410)$ should have a node in its wave function, which would naturally translate into a node in the decay amplitude. A nodal Gaussian form factor (Eq. 3) is accordingly found in the phenomenologically successful ${ }^{3} P_{0}$ model; for the decay to $D \pi, D^{*} \pi$ of all interpretations of $D(2637)$ discussed in the previous section, except $D_{3}^{*}$ and $D_{2} j_{q}=\frac{5}{2}$. For these interpretations we perform a search for decays to $D \pi$ and $D^{*} \pi$ consistent with the DELPHI bound, using the methods of the previous section. Only successful searches are highlighted.

$D^{\prime}$, using $K^{*}(1410)$

Using Eqs. \& and 3, the ratio $\Gamma\left(K^{*}(1410) \rightarrow K \pi\right) / \Gamma\left(K^{*}(1410) \rightarrow K^{*} \pi\right)$ and width $\Gamma\left(K^{*}(1410) \rightarrow K \pi\right)$ from experiment $[7,20]$, we determine $F_{11}^{\frac{1}{2} \frac{1}{2}}(0)$ and $0.21 \leq \tilde{\beta} \leq 0.25$ $\mathrm{GeV}$, where the two extrema of the range has the advantage that they allow consistency with other experimental datal. From Eqs. 1 and 3 we estimate $\Gamma\left(D^{\prime} \rightarrow D^{*} \pi\right)$, which is substantial $(\sim 100 \mathrm{MeV})$ for most of the allowed $\tilde{\beta}$ range. However, for the lower extremum $\Gamma\left(D^{\prime} \rightarrow D^{*} \pi\right)$ is as low as $10 \mathrm{MeV}$. Assuming flavour $\mathrm{SU}(3)$ symmetry, we can also estimate $\Gamma\left(D^{\prime} \rightarrow D^{*} \eta\right)=20,26 \mathrm{MeV}$ at the lower extremum 21]. Fixing from $\Gamma\left(K^{*}(1410) \rightarrow K \pi\right)$ we find that the sum of the decays to $D^{*} \pi, D^{*} \eta$ and $D_{s}^{*} K$ can be as low as $7.9 \mathrm{MeV}$ and consistent with the DELPHI total width for $0.13 \leq \tilde{\beta} \leq 0.20$, a region that is disjoint,

\footnotetext{
${ }^{1}$ Also, the heavy quark symmetry prediction for $\Gamma\left(K^{*}(1410) \rightarrow K \pi\right) / \Gamma\left(K(1460) \rightarrow K^{*} \pi\right)$ with a Gaussian form factor is five times larger than experiment.

${ }^{2} \Gamma\left(K^{*}(1410) \rightarrow K \pi\right) / \Gamma\left(K(1460) \rightarrow K^{*} \pi\right)=0.13,0.11$ for $\tilde{\beta}=0.21,0.25 \mathrm{GeV}$ respectively, versus an experimental value of 0.14 20].
} 
but tantalizingly close, to the preferred region $0.21 \leq \tilde{\beta} \leq 0.25 \mathrm{GeV}$. $D^{\prime}$ should hence be considered too wide to be in agreement with the DELPHI width, although this depends sensitively on the experimental data on $K^{*}(1410) \rightarrow K \pi, K^{*} \pi$.

\section{$D^{\prime}$, using $K^{*}(1680)$}

The ratio $\Gamma\left(K^{*}(1680) \rightarrow K \pi\right) / \Gamma\left(K^{*}(1680) \rightarrow K^{*} \pi\right)$ is $1.30_{-0.14}^{+0.23}$ (or $2.8 \pm 1.1$ directly from the LASS data) [7]. This ratio, together with $\Gamma\left(K^{*}(1680) \rightarrow K \pi\right)$ [20] is used to estimate $\Gamma\left(D^{\prime} \rightarrow D^{*} \pi\right)$, and within $\mathrm{SU}(3)$ symmetry $\Gamma\left(D^{\prime} \rightarrow D^{*} \eta, D_{s}^{*} K\right)$. We are able to find a total $D^{*} \pi, D^{*} \eta$ and $D_{s}^{*} K$ width of less than $15 \mathrm{MeV}$ only when we assume $\Gamma\left(K^{*}(1410) \rightarrow K \pi\right) / \Gamma\left(K^{*}(1410) \rightarrow K^{*} \pi\right) \geq 3.4$, consistent, but at the very edge of the LASS error barsf. Consistency with the DELPHI bound is hence unnatural, but can be achieved.

$D_{1 j_{q}=\frac{1}{2}}^{*}$, using $K_{0}^{*}(1430)$ and $K_{1}(1400)$

$K_{1}(1400)$ is interpreted as the heavy quark symmetry partner of the $K_{0}^{*}(1430)$, based on the D- to S-wave width ratio and the interpretation of $K_{1}(1270)$ as the $j_{q}=\frac{3}{2}$ state [5, 6, 4].

Fixing from the ratio $\Gamma\left(K_{0}^{*}(1430) \rightarrow K \pi\right) / \Gamma\left(K_{1}(1400) \rightarrow K^{*} \pi\right)$ and $\Gamma\left(K_{0}^{*}(1430) \rightarrow K \pi\right.$ 20] we obtain a solution with $\tilde{\beta}=0.19 \mathrm{GeV}$ and $\Gamma\left(D_{1 j_{q}=\frac{1}{2}}^{*} \rightarrow D^{*} \pi\right)=13 \mathrm{MeV}$. This is remarkably narrow and due to the amplitude "hitting a node". Within SU(3) we estimate $\Gamma\left(D_{1 j_{q}=\frac{1}{2}}^{*} \rightarrow D^{*} \eta\right)=67-90 \mathrm{MeV}$ [21]. The total width of $D_{1 j_{q}=\frac{1}{2}}^{*}$ is hence inconsistent with the DELPHI value.

In conclusion, except for $D_{3}^{*}$ and $D_{2} j_{q}=\frac{5}{2}$ which are not assumed to have a nodal form factor, all interpretations of $D(2637)$ have too large a width, except possibly $D^{\prime}$. This conclusion is contingent on our inability to calculate $D^{\prime}$ decays to $D_{0}^{*} \pi, D_{2 j_{q}=\frac{5}{2}} \pi, D(\pi \pi)_{S}$ and $D \rho$ from heavy quark symmetrył.

\footnotetext{
${ }^{3}$ At the edge we obtain a $D^{*} \pi$ width as low as $9.7 \mathrm{MeV}$. The solutions have $\tilde{\beta}=0.15-0.16 \mathrm{GeV}$.

${ }^{4}$ Decays to $D_{0}^{*} \pi, D \rho$ and $D^{*} \rho$ can only be estimated from current data on $K(1460)$, the existence of which is controversial. Particularly, $\Gamma\left(K(1460) \rightarrow K_{0}^{*}(1430) \pi\right)=177 \mathrm{MeV}$ [7] is a substantial S-wave decay below threshold; and should induce a huge $D^{\prime} \rightarrow D_{0}^{*} \pi$ width since this decay is slightly above threshold for the $D_{0}^{*}$ mass of ref. [9].
} 


\section{Determining the $J^{P}$ of $D(2637)$ from $\mathbf{K}$-meson masses}

DELPHI made the preliminary claim of an enhancement atf $5905 \pm 11 \mathrm{MeV}$ decaying to $B^{*} \pi^{+} \pi^{-}$[22]. Given the similarity of this decay mode to the observation of $D(2637) \rightarrow$ $D^{*} \pi^{+} \pi^{-}[1]$, we postulate that $B(5905)$ and $D(2637)$ are analogues of each other with different heavy quarks, and explore the consequences.

Up to $1 / m_{Q}$ corrections to heavy quark symmetry, we can write for the mass of the heavylight meson $B(5905)$ and $D(2637)$ [5, 6]

$$
M(B(5905))=M(1 S)_{B}+E+\frac{C}{m_{b}} \quad M(D(2637))=M(1 S)_{D}+E+\frac{C}{m_{c}}
$$

where e.g. $M(1 S)_{B}=\left(3 M\left(B^{*}\right)+M(B)\right) / 4$ is the mass of the ground state. The efficacy of using the approach in Eq. 4 to estimate heavy-light meson masses is seen by noting that, the predictions of this approach for $D_{s J}(2573), D_{s 1}(2536), B_{J}^{*}(5732)$ and $B_{s J}^{*}(5850)$ [5, 6] are in as good agreement with experiment as potential models [8, 9, 11]. The first set of charm and botton quark masses is taken to be $m_{c}=1.48 \mathrm{GeV}, m_{b}=4.8 \mathrm{GeV}$; and the second set $m_{c}=1.84 \mathrm{GeV}, m_{b}=5.18 \mathrm{GeV}$, following refs. [5, 6]. These two sets of masses include most of the range found in potential models, particularly those of refs. [8, 9, 16, 19. Using the analogous equations to Eq. \$ 4 and following refs. [5, 6] by fitting $K_{2}^{*}(1430)$, $K_{1}(1270), D_{2}^{*}(2460)$ and $D_{1}(2420)$ according to the newest PDG masses [7], one obtains $m_{s}=0.348 \mathrm{GeV}$ for set one and $m_{s}=0.433 \mathrm{GeV}$ for set two.

There are two equations in Eq. 田, which we solve for the two unknowns $E$ and $C$. Substituting these values into the expression for the mass of the $\mathrm{K}$-meson analogue of $B(5905)$ and $D(2637), M(1 S)_{K}+E+\frac{C}{m_{s}}$, we obtain the $\mathrm{K}$-meson mass $1820 \pm 60 \mathrm{MeV}$ and $1850 \pm 70$ $\mathrm{MeV}$ for the first and second sets of $m_{s}, m_{c}$ and $m_{b}$ respectively.

In conclusion, assuming the validity of the masses of $B(5905)$ and $D(2637)$ from experiment, and that they are simply analogues of each other with different heavy quarks, the lowest order correction to heavy quark symmetry predicts that the $\mathrm{K}$-meson analogue should have a mass of $1800 \pm 60 \mathrm{MeV}$ or $1820 \pm 70 \mathrm{MeV} . K_{3}^{*}(1780), K_{2}(1770)$ and $K_{2}(1820)$ are comfortably within, and $K^{*}(1680)$ at the edge of, these mass regions. Given that $D(2637)$

\footnotetext{
${ }^{5}$ Obtained from the datum $301 \pm 4 \pm 10 \mathrm{MeV}=M\left(B^{*} \pi^{+} \pi^{-}\right)-M\left(B^{*}\right)-2 M(\pi)$ 22]. We have ignored the possibility that decay of the enhancement to $B \pi^{+} \pi^{-}$is also allowed by the data [22].
} 
is an analogue of one of these states, the $J^{P}$ of $D(2637)$ is $2^{-}, 3^{-}$or possibly $1^{-}$.

\section{Comments and conclusions}

It is critical to corroborate the claim by DELPHI of such a small $D(2637)$ total width. The total width is more discriminatory than individual partial widths, e.g. with the nodal

form factor the decay $D_{1 j_{q}=\frac{1}{2}}^{*} \rightarrow D^{*} \pi$ is small, but the collective decay to $D^{*} \pi$ and $D^{*} \eta$ is substantial. Dominant modes are likely to be $D \pi, D^{*} \pi, D^{*}(\pi \pi)_{S}$ and for some interpretations $D(\pi \pi)_{S}$. A small width for $D(2637)$ would put a restrictive bound on $D^{*}(\pi \pi)_{S}$, and for some interpretations on $D(\pi \pi)_{S}$. This would be a useful input into models. The $J^{P}$ of $D(2637)$ can experimentally be ascertained without partial wave analysis. For example, of the possibilities considered only $0^{-}$and $2^{-}$should have enough phase space for the experimentally challenging decay to $D_{0}^{*} \pi$. Only $1^{+}, 0^{-}$and $2^{-}$decay to $D(\pi \pi)_{S}$ and do not decay to $D \pi, D \eta$ and $D_{s} K$.

If the DELPHI mass and width of $D(2637)$ are confirmed, it would present a fascinating challenge for theory. Within heavy quark symmetry, the width cannot be explained by an exhaustive list of possibilities, except possibly if the state is $D_{3}^{*}, D_{2} j_{q}=\frac{5}{2}$ or $D^{\prime}$. However, these possibilities are inconsistent with potential model mass estimates. Moreover, if $D(2637)$ is either $D_{3}^{*}$ or $D_{2} j_{q}=\frac{5}{2}$ then it appears that the other (unobserved) resonance should appear within $20 \mathrm{MeV}$ of it [8]. The interpretation of $D(2637)$ as $D^{\prime}$ is complicated by the fact that decay via the kinematically preferred route $D^{*}(\pi \pi)_{S}$ is not allowed. Since $D(2637)$ is observed in $D^{*} \pi \pi$, this would have to arise via kinematically suppressed routes like $D^{*} \rho$ and $D_{2}^{*}(2460) \pi$. Of the potentially narrow interpretations of $D(2637), D_{3}^{*}$ and $D_{2 j_{q}=\frac{5}{2}}$ are preferred when the implications of the lowest order corrections to heavy quark symmetry on heavy-light meson masses are analysed. If one insists that potential model mass calculations are correct, $D(2637)$ must be $D^{*^{\prime}}$, and we speculate that complicated nodal dynamics may give rise to the experimental width. This may serve as a sensitive probe of detailed decay dynamics, yielding tantalizing insight into the pair creation mechanism, e.g. ${ }^{3} P_{0}$ pair creation [16, 17, 23].

\section{References}


[1] P. Abreu et al. (DELPHI Collab.), Phys. Lett. B426 (1998) 231; C. Bourdarios (DELPHI Collab.), Proc. of $29^{\text {th }}$ Int. Conf. on High Energy Physics (ICHEP'98) (Vancouver, Canada, July 1998), to be published by World Scientific, Singapore.

[2] I. Shipley (CLEO Collab.), P. Krieger (OPAL Collab.), Proc. of 29 ${ }^{\text {th }}$ Int. Conf. on High Energy Physics (ICHEP'98) (Vancouver, Canada, July 1998), to be published by World Scientific, Singapore.

[3] For a discussion consult refs. [7, 18] and N.A. Törnqvist, Nucl. Phys. Proc. Suppl. B21 (1991) 196.

[4] M. Suzuki, Phys. Rev. D50 (1994) 4708; T. Ito, T. Morii, and M. Tanimoto, Prog. Theor. Phys. 90 (1993) 377; N. Isgur, Proc. of Baryon'96 (Santa Fe, NM) and Baryon'98 (Bonn, Germany).

[5] E.J. Eichten, C.T. Hill, C. Quigg, Phys. Rev. Lett. 71 (1993) 4116.

[6] E.J. Eichten, C.T. Hill, C. Quigg, FERMILAB-CONF-94-118-T.

[7] C. Caso et al. (Particle Data Group), Eur. Phys. J. C3 (1998) 1.

[8] S. Godfrey, N. Isgur, Phys. Rev. D32 (1985) 189.

[9] D. Ebert, R.N. Faustov, V.O. Galkin, Phys. Rev. D57 (1998) 5663; Proc. of $10^{\text {th }}$ Int. Seminar on High Energy Physics (Quarks'98) (Suzdal, Russia, May 1998).

[10] A mass of $2.461 \mathrm{GeV}$ is reported by J. Rodriguez, Proc. of $4^{\text {th }}$ Workshop on Heavy Quarks at Fixed Target (Heavy Quark '98) (Batavia, IL, Oct. 1998).

[11] N. Isgur, Phys. Rev. D57 (1998) 4041.

[12] A.B. Kaidalov, A.V. Nogteva, Sov. J. Nucl. Phys. 47 (1988) 321.

[13] N. Isgur, M.B. Wise, Phys. Rev. Lett. 66 (1991) 1130; A.F. Falk, M. Luke, Phys. Lett. B292 (1992) 119.

[14] D.A. Varshalovich, A.N. Moskalev, V.K. Khersonskii, "Quantum Theory of Angular Momentum", World Scientific, Singapore, 1988.

[15] S. Godfrey, R. Kokoski, Phys. Rev. D43 (1991) 1679. 
[16] R. Kokoski, N. Isgur, Phys. Rev. D 35 (1987) 907.

[17] T. Barnes, F.E. Close, P.R. Page, E.S. Swanson, Phys. Rev. D55 (1997) 4157; A. Le Yaouanc, L. Oliver, O. Péne, J.-C. Raynal, "Hadron Transitions in the Quark Model", Gordon and Breach Science Publishers S.A., New York, 1988.

[18] L. Burakovsky, T. Goldman, Nucl. Phys. A625 (1997) 220.

[19] E.J. Eichten, C.T. Hill, C. Quigg, FERMILAB-CONF-94-117-T.

[20] Deduced using the experimental branching ratios and total width [7].

[21] For $\eta=\frac{1}{\sqrt{2}}\left(\frac{1}{\sqrt{2}}(u \bar{u}+d \bar{d})-s \bar{s}\right)$ or $\frac{1}{\sqrt{3}}(u \bar{u}+d \bar{d}-s \bar{s})$ respectively.

[22] M. Feindt, O. Podobrin, "First Observation of Radially Excited B Mesons", contribution to $28^{\text {th }}$ Int. Conf. on High Energy Physics (ICHEP'96) (Warsaw, Poland, June 1996), ref. pa01-021, DELPHI 96-93 CONF 22.

[23] F.E. Close, P.R. Page, Phys. Rev. D56 (1997) 1584. 